\title{
Verifications of a formal technique for viscoelastodynamics
}

\section{D.W. Barclay and T. Bryant Moodie}

\begin{abstract}
Presented in this paper are justifications for the formal KaralKeller technique as it applies to propagation, reflection, and transmission of one-dimensional impact waves in nonhomogeneous viscoelastic solids.
\end{abstract}

\section{Introduction}

From 1944 to the present, a formal asymptotic technique, based on generalizations of geometrical optics, has been developed and applied to various areas of mathematical physics. Herein we will explain this method and provide some rigorous justifications for its validity as applied to waves in nonhomogeneous hyperbolic-viscoelastic materials.

In a series of lectures [9] given at Brown University in the summer of 1944, Luneburg presented an approach to the problem of reconciling geometrical optics to wave optics. He constructed an asymptotic expansion in inverse powers of the frequency for the solution of Maxwell's equation for a time-harmonic field and demonstrated that the leading term of this expansion is the geometrical optics solution. Several authors (see [7] for a list of references) have shown that subsequent terms account for diffraction effects, that is, effects which cannot be accounted for by classical geometrical optics. Friedlander [3] formulated Luneburg's technique so that it applies to general progressing waves.

Karal and Keller [4], using Friedlander's formulation to bypass the often difficult Fourier synthesis, extended Luneburg's technique to study

Received 1 June 1974. 
general progressing waves in isotropic elastic media. We will refer to this formal asymptotic technique, when applied to waves in solid media, as the Karal-Keller technique. Let us indicate briefly the steps involved in the Karal-Keller asymptotic method. For problems whieh can be solved exactly, examination of the asymptotic expansion of the solution shows that it consists of a sum of terms each of which is an asymptotic series involving a phase function and an infinite sequence of amplitude functions. For complex problems it is assumed that the solution is also a sum of such series. Inserting the series into the governing partial differential equation one finds that the phase and amplitude functions satisfy ordinary differential equations along the rays associated with the wave motion. For some problems the initial conditions for all of these ordinary differential equations can be found directly from the data of the problem ([1]) while for others one must resort to the device of canonical problems and the 'principle of localization' [8].

It is clear that the Karal-Keller asymptotic method involves several unproved assumptions. It is therefore reasonable to ask whether it can be proved that it does indeed yield the asymptotic expansion of the exact solution for the given problem. No general proof of this fact has been published. Evidence for the method's validity can be obtained in one of the following ways:

(i) comparisons can be made of results obtained using the Karal-Keller method and the asymptotic expansion of known exact solutions,

(ii) comparisons with experimental and numerical methods can be carried out, or

(iii) in the case of oscillatory initial data, energy inequalities can be used [6] to estimate the remainder of the asymptotic series.

In connection with the above for elastodynamics, we mention a paper by Lang [5]. He applied the Karal-Keller theory to the problem of a spherical source in homogeneous elastic media and demonstrated that the displacement series terminates after two terms to yield the known exact solution. Cooper [1] demonstrated that, for one-dimensional longitudinal waves in a particular nonhomogeneous half-space, the Karal-Keller technique generates 
a terminating series which is the known exact solution. One of the present authors [10], applying the Karal-Keller technique to the two-dimensional problem of an impulsive source of cylindrical shear waves in media whose shear modulus is proportional to an arbitrary power of radial distance from the axis of symmetry, obtained complete agreement with known exact solutions.

In the field of viscoelastodynamics, the Karal-Keller techrique has been applied to one-dimensional waves in a standard nonhomogeneous rod by Park and Reiss [13]. These authors provided a partial justification for the method as it applies to waves in a semi-infinite homogeneous rod by demonstrating that the leading term of the exact and formal solutions are in agreement. Moodie [11], [12] applied the method to several problems involving cylindrical and spherical shear waves in nonhomogeneous media. Because we feel that the formal Karal-Keller asymptotic technique offers a viable method for solving problems involving dynamical loading of nonhomogeneous viscoelastic materials, we will here present justification for its use. This we shall do by showing that, for one-dimensional waves in nonhomogeneous viscoelastic rods, the Karal-Keller technique gives asymptotic results which are in agreement with those obtained from the exact solution. We shall also demonstrate that for reflection and transmission, the results obtained by this method are in agreement with those obtained rigorously from the exact solution. We thus go much further than did Park and Reiss in providing a justification for the Karal-Keller technique.

\section{Formulation}

We choose the centre line of a standard viscoelastic rod to coincide with the positive $x$-axis. According to the one-dimensional theory of longitudinal waves in rods, the equation of motion of the rod is

$$
\sigma_{x}=\rho u_{t t}
$$

where $u(x, t)$ is the axial displacement, $\sigma(x, t)$ the axial stress, and $\rho(x)$ the prescribed density. The literal subscripts refer to differentiation with respect to the corresponding variable. The constitutive equation for the standard viscoelastic solid is 


$$
\sigma_{t}+\left(E_{1} / \eta\right) \sigma=\left(E+E_{1}\right) u_{x t}+\left(E E_{1} / \eta\right) u_{x} \text {, }
$$

where $E(x)$ and $E_{1}(x)$ are elastic moduli, and $\eta(x)$ is a viscous modulus. Combining (1) and (2), the stress equation of motion of the rod is

$$
(\partial / \partial t)\left[\sigma_{t t}-\gamma^{2} \sigma_{x x}+\gamma^{2} r \sigma_{x}\right]+\lambda\left[\sigma_{t t}-c^{2} \sigma_{x x}+c^{2} r \sigma_{x}\right]=0,
$$

where the coefficients are defined by

(4) $\gamma^{2}(x)=\left(E+E_{1}\right) / \rho, e^{2}(x)=E / \rho, r(x)=\rho_{x} / \rho, \lambda(x)=E_{1} / n$.

The dimensions of $\gamma$ and $c$ are that of velocity.

We now assume [3] that the stress solution may be represented by

$$
\sigma \sim \sum_{n=0}^{\infty} A_{n}(x) F_{n}[t-S(x)], A_{n} \equiv 0 \text { for } n<0 \text {, }
$$

where the $F_{n}^{\prime}$ s are related by

$$
F_{n}^{\prime}=F_{n+1}, n=1,2, \ldots .
$$

We further assume that the derivatives of $\sigma$ may be obtained by term-wise differentiation in (5). The prime in (6) denotes differentiation with respect to the entire argument $t-S$ and $(6)$ enables us to relate all of the $F_{n}^{\prime}$ 's to $F_{0}$ (the waveform) by successive integrations. For example, if $F_{0}$ is the Heaviside step function, $H(t)$, defined by

$$
H(t)=1 \text { for } t>0, H(t)=0 \text { for } t<0 \text {, }
$$

then

$$
F_{n}=\frac{(t-S)^{n}}{n !} H(t-S)
$$

Note that $F_{0}$ vanishes for negative argument, that is, in front of the wavefront whose equation is given by $t=S(x)$, where $S$ is called the phase or eikonal function. For $F_{n}$ given by (7), the coefficients $A_{n}$ in (5) are the jump conditions for the stress and its derivatives across the wavefront. Solutions of (3) in the form (5) offer a convenient device for studying the propagation of stress discontinuities. However, it should be 
emphasized that solutions of this form are applicable only for hyperbolicviscoelastic materials, that is, materials in which discontinuities propagate with finite speed.

\section{The general Karal-Keller solution}

We consider longitudinal waves whose propagation is governed by equation (3) and seek the stress solution in the form (5). Substituting (5) into (3) and using the arguments outlined in [10], we derive

$$
\left(S^{\prime}\right)^{2}=[\gamma(x)]^{-2}
$$

and

$$
2 S^{\prime} A_{n}^{\prime}+S^{\prime \prime} A_{n}+\lambda\left[1-c^{2}\left(S^{\prime}\right)^{2}\right] A_{n} / \gamma^{2}-r S^{\prime} A_{n}=R_{n}, \quad n \geq 0
$$

where

$$
\text { (10) } \begin{aligned}
R_{n} \equiv A_{n-1}^{\prime \prime}-r A_{n-1}^{\prime}-2 \lambda c^{2} S^{\prime} A_{n-1}^{\prime} / \gamma^{2}+\lambda c^{2} r S^{\prime} A_{n-1} / \gamma^{2} \\
-\lambda c^{2} S^{\prime \prime} A_{n-1} / \gamma^{2}+\lambda c^{2} A_{n-2}^{\prime \prime} / \gamma^{2}-\lambda c^{2} r A_{n-2}^{\prime} / \gamma^{2} .
\end{aligned}
$$

Equation (8) is the well-known eikonal equation of geometrical optics. Along the rays associated with the longitudinal disturbance it becomes an ordinary differential equation which may be integrated to give

$$
S(x)=\bar{S} \pm \int_{\bar{x}}^{x} d \tau / \curlyvee(\tau), \bar{S} \equiv S(\bar{x})
$$

The plus sign corresponds to waves propagating in the positive $x$ direction while the minus sign corresponds to waves propagating in the negative $x$ direction. Equation (11) enables us to determine the phase at any point on a ray in terms of its value at $x=\bar{x}$.

Equation (9) is the so called transport equation for determining the amplitude functions $A_{n}$. Its general solution is 
(12) $A_{n}(x)=\bar{A}_{n}\left[\frac{\gamma(x) \rho(x)}{\bar{\gamma} \bar{\rho}}\right]^{\frac{3}{2}} \exp \left\{\mp \frac{1}{2} \int_{\bar{x}}^{x} \frac{\lambda(\tau)}{\gamma(\lambda)}\left[1-\frac{c^{2}(\tau)}{\gamma^{2}(\tau)}\right] d \tau\right\}$

$$
\begin{gathered}
\pm \frac{1}{2} \int_{\bar{x}}^{x} \gamma(\tau)\left[\frac{\gamma(x) \rho(x)}{\gamma(\tau) \rho(\tau)}\right]^{\frac{2}{2}} \exp \left\{\mp \frac{1}{2} \int_{\tau}^{x} \frac{\lambda(y)}{\gamma(y)}\left[1-\frac{c^{2}(y)}{\gamma^{2}(y)}\right] d y\right\} R_{n}(\tau) d \tau, \\
\bar{A}_{n} \equiv A_{n}(\bar{x}), \bar{\gamma} \equiv \gamma(\bar{x}), \bar{\rho} \equiv \rho(\bar{x}),
\end{gathered}
$$

where the upper signs correspond to waves propagating in the direction of positive $x$ and the lower signs to waves propagating in the direction of negative $x$. In (12) it is very important to remember and choose the correct sign for $S$ in $R_{n}$ - thus accounting for the direction of propagation.

\section{Karal-Keller solutions to particular problems}

In this section we will examine some particular impact problems by means of the Karal-Keller asymptotic technique.

PROBLEM 1. The semi-infinite rod $x \geq 0$, defined by the properties

$$
\begin{gathered}
c^{2}(x)=a \gamma^{2}(x), 0<a<1, \\
\lambda=\lambda_{0}, r=r_{0} /(1+x), r_{0}>0, \\
\gamma^{2}=\gamma_{0}^{2}(1+x)^{2}, \gamma_{0}>0,
\end{gathered}
$$

where $a, \lambda_{0}, r_{0}$ and $\gamma_{0}$ are constants, is initially unstressed and at rest. For $t>0$, a pressure is applied to the end $x=0$, so that $\sigma(0, t)$ is known. The solution will first be obtained for a step pressure input, and Duhamel's Theorem will be applied to obtain the solution for a general input pressure function. Thus, from (5),

$$
\sigma(0, t) \sim \sum_{n=0}^{\infty} \bar{A}_{n} F_{n}[t-\bar{S}]=-H(t),
$$

where bars indicate that the variables are evaluated at $x=\bar{x}=0$. Hence, we choose 
(15)

$$
\begin{aligned}
& \bar{A}_{n}=\left\{\begin{array}{l}
-1 \text { if } n=0, \\
0 \text { if } n>0 \text { or } n<0,
\end{array}\right. \\
& \bar{s}=0, F_{0}=H(t) \text {. }
\end{aligned}
$$

Employing (13) and (15) in (11) (taken with the plus sign so as to correspond to a wave travelling in the direction of positive $x$ ) we obtain for the phase $S$,

$$
S(x)=[\ln (1+x)] / \gamma_{0}
$$

Substituting from (13) into (12) (taken with the upper signs) it is easy to prove by mathematical induction that

$$
A_{n}=(1+x)^{\varepsilon} \sum_{j=0}^{n} a_{j n}[\ln (1+x)]^{j}
$$

where

$$
\varepsilon \equiv\left[\gamma_{0}\left(1+r_{0}\right)-\lambda_{0}(1-a)\right] / 2 \gamma_{0}
$$

Substituting (17) into (12) and performing various simplifications, we obtain

$$
\text { (19) } a_{j n}=\left\{\begin{aligned}
& \frac{\gamma_{0}}{2}\left\{\lambda_{0} a(j+1) a_{j+1, n-2}+(j+1) a_{j+1, n-1}+\lambda_{0} a\left[2 \varepsilon-1-r_{0}\right] a_{j, n-2}\right. \\
&+\left[(2 \varepsilon-1)-r_{0}-2 \lambda_{0} a / \gamma_{0}\right] a_{j, n-1}+\lambda_{0} a \varepsilon\left[\varepsilon-1-r_{0}\right] a_{j-1, n-2} / j \\
&\left.+\left[\varepsilon(\varepsilon-1)-\varepsilon\left(r_{0}+2 \lambda_{0} a / \gamma_{0}\right)+\lambda_{0} a\left(1+r_{0}\right) / \gamma_{0}\right] a_{j-1, n-1} / j\right\} \\
& \text { for } 1 \leq j \leq n, \\
&-1 \text { if } j=n=0, \text { or } j>n,
\end{aligned}\right.
$$

where $a_{j, n} \equiv a_{j n}$ and the boundary conditions (15) have been used in deriving (19).

The complete wavefront expansion of the solution to the above impact problem is then given by

$$
\sigma \sim(1+x)^{\varepsilon} \sum_{n=0}^{\infty} \frac{(t-S)^{n}}{n !} \sum_{j=0}^{n} a_{j n}[\ln (1+x)]^{j} H(t-S),
$$


where $S$ is given by (16) and the $a_{j n}$ are given recursively by (19). If we evaluate the first three terms of our expression for $\sigma$ given by $(20)$, we have

(21) $\sigma \sim(1+x)^{\varepsilon}\left\{-1+\frac{B_{1} \ln (x+1)}{\gamma_{0}}(t-S)-\frac{1}{2}\left[\frac{1}{2}\left[\frac{\beta_{1} \ln (x+1)}{\gamma_{0}}\right]^{2}\right.\right.$ $\left.\left.-\frac{\beta_{2} \ln (x+1)}{\gamma_{0}}\right](t-S)^{2}\right\} H(t-S)$,

where

$$
\begin{aligned}
& \beta_{1}=-\frac{\gamma_{0}^{2}}{2}\left[\varepsilon(\varepsilon-1)-\varepsilon\left(r_{0}+\frac{2 \lambda_{0} a}{\gamma_{0}}\right)+\frac{\lambda_{0} a\left(r_{0}+1\right)}{\gamma_{0}}\right], \\
& \beta_{2}=-\frac{\lambda_{0}}{2}(a+1) \beta_{1}+\frac{\lambda_{0} a}{8}\left[\left(r_{0}+1\right)^{2} \gamma_{0}^{2}-(a-1)^{2} \lambda_{0}^{2}\right] .
\end{aligned}
$$

Later we will show that this expansion agrees with the asymptotic expansion rigorously derived from the exact solution.

In general the solution for an arbitrary input pressure, $\sigma(0, t)=f(t)$, may be obtained from Duhamel's Theorem,

$$
\sigma(x, i)=\int_{0}^{t} \frac{\partial \sigma^{*}}{\partial \tau}(x, \tau) f(t-\tau) d \tau
$$

where $\sigma^{*}$ is the solution for a step input compressive stress at $x=0$, that is, $\sigma^{*}$ is given by (20). If, for example, $f(t)=-\delta(t)$, where $\delta(t)$ is the Dirac delta function, then

$$
\sigma \sim-(1+x)^{\varepsilon} \delta(t-S)+(1+x)^{\varepsilon} \sum_{n=1}^{\infty} \frac{(t-S)^{n}}{n !} \sum_{j=0}^{n} a_{j n}[\ln (1+x)]^{j} H(t-S)
$$

where $S$ is given by (16) and the $a_{j n}$ by (19).

We should here emphasize that although the solution has been obtained for the particular viscoelastic material defined by (13), we could have obtained the solution $\sigma$ for many very general classes of materials. However, as we wish to effect a comparison between results obtained by the Karal-Keller technique and the exact solution, we are restricted to those 
materials for which the exact solution may be obtained - this being a very restrictive class. The power of the Karal-Keller technique resides in the fact that it enables one to solve problems not amenable to classical boundary value procedures. In fact, the Karal-Keller technique does not rely on separability.

PROBLEM 2. We shall now, for completeness, outline briefly the procedure for treating the impact problem for a finite rod $0 \leq x \leq L$. We choose the condition at $x=\bar{x}=L$ to be that of vanishing stress, that is,

$$
\bar{\sigma}=0
$$

Other boundary conditions than (25) can be handled by the methods presented here. We consider this finite rod to be initially unstressed and at rest. For $t>0$, a step compressive pressure is applied at the end $x=0$, that is,

$$
\bar{\sigma}=-H(t), \quad x=\bar{x}=0 .
$$

A transient stress wave is produced which leaves the end $x=0$ at $t=0^{\circ}$ and travels with variable velocity $\gamma(x)$ in the direction of positive $x$. This outgoing wave has been discussed above and its wavefront asymptotic expansion is given by (5) where the $F_{n}, S$, and $A_{n}$ are determined from (7), (11), and (12) together with the appropriate boundary conditions (15). The upper signs in (11) and (12) must be chosen so that this expansion corresponds to a wave which is outgoing with respect to the source at $x=0$. From now on we will denote this outgoing wave by $\sigma^{l}$ and its amplitude and phase functions by $A_{n}^{l}$ and $S^{1}$. The leading term in the expansion for the outgoing wave is found to be

$$
\sigma^{I} \sim-\left[\frac{\gamma(x) \rho(x)}{\gamma(0) \rho(0)}\right]^{\frac{3}{2}} \exp \left\{-\frac{1}{2} \int_{0}^{x} \frac{\lambda(\tau)}{\gamma(\tau)}\left[1-\frac{c^{2}(\tau)}{\gamma^{2}(\tau)}\right] d \tau\right\} H\left(t-S^{1}\right)
$$

where

$$
S^{1}=\int_{0}^{x} \frac{d \tau}{\gamma(\tau)} \text {. }
$$

We now assume that at the stress-free boundary there is produced a 
reflected wave, $\sigma^{2}$, which can be represented by

$$
\sigma^{2} \sim \sum_{n=0}^{\infty} A_{n}^{2}(x) F_{n}\left[t-S^{2}(x)\right], A_{n}^{2} \equiv 0 \text { for } n<0 .
$$

The amplitude functions, $A_{n}^{2}$, satisfy the transport equation (9) and the phase function, $S^{2}$, satisfies the eikonal equation (8). The solution of (10), for a wave travelling from $x=L$ in the direction of decreasing $\boldsymbol{x}$, is given by (12) taken with the lower signs and $\bar{x}=L$. The phase function, $S^{2}$, is given by (1I) taken with the minus sign and $\bar{x}=L$. We justify our assumption that a reflected wave is produced at $x=L$ by demonstrating that the boundary condition (25) can be satisfied by

$$
\sigma=\sigma^{1}+\sigma^{2} \text {. }
$$

Up until time $t=S^{l}(L)$ the solution is given by the incident wave $\sigma^{\mathcal{I}}$, which has been completely determined above. At $t=S^{1}(L)$ the incident wave has arrived at the boundary $x=\bar{x}=L$. Applying the boundary condition of vanishing stress we obtain

$$
\vec{A}_{n}^{2}=-\bar{A}_{n}^{I}, \bar{S}^{2}=\bar{S}^{I}, \quad x=\bar{x}=L .
$$

Then, because $\bar{A}_{n}^{1}$ and $\bar{S}^{1}$ are known, we may completely determine the reflected wave, $\sigma^{2}(x, t)$, by the methods employed above. To a first term approximation this reflected wave is given by

(32) $\sigma^{2} \sim\left[\frac{\gamma(x) \rho(x)}{\gamma(0) \rho(0)}\right]^{\frac{1}{2}} \exp \left\{-\frac{1}{2}\left(\int_{0}^{L}+\int_{x}^{L}\right) \frac{\lambda(\tau)}{\gamma(\tau)}\left[1-\frac{c^{2}(\tau)}{\gamma^{2}(\tau)}\right] d \tau\right\} H\left(t-S^{2}\right)$,

where

$$
S^{2}=\left(\int_{0}^{L}+\int_{x}^{L}\right) \frac{d \tau}{\gamma(\tau)}
$$

PROBLEM 3. In this our final application of the Karal-Keller technique, we consider the impact problem for a step compressive pressure applied to the end of a semi-infinite nonhomogeneous viscoelastic bar composed of two media initially unstressed and at rest. These media we 
label medium " $a$ " and medium " $b$ ". We denote the compressional wave speed $\gamma$ and density $\rho$ of the two media by $\gamma_{a}, \gamma_{b}, \rho_{a}$, and $\rho_{b}$ with a similar notation for the elastic and viscous moduli. A transient stress wave is produced which leaves the end $x=0$ at $t=0$ and travels with variable velocity $\gamma_{a}(x)$ in the direction of positive $x$ towards the interface at $x=\bar{x}=L$. The boundary conditions at the interface require that $\bar{\sigma}_{a}=\bar{\sigma}_{b}$ and $\bar{v}_{a}=\bar{v}_{b}$, where $v(x, t)$ is the particle velocity. We assume that a wave is reflected by the boundary and that a wave is transmitted across the boundary. Thus, we assume

$$
\sigma_{\alpha} \sim \sum_{n=0}^{\infty} A_{n}^{1} F_{n}\left(t-S^{1}\right)+\sum_{n=0}^{\infty} A_{n}^{2} F_{n}\left(t-S^{2}\right),
$$

$$
\begin{aligned}
& \sigma_{b} \sim \sum_{n=0}^{\infty} A_{n}^{3} F_{n}\left(t-S^{3}\right), \\
& v_{a} \sim \sum_{n=0}^{\infty} B_{n}^{1} F_{n}\left(t-S^{1}\right)+\sum_{n=0}^{\infty} B_{n}^{2} F_{n}\left(t-S^{2}\right),
\end{aligned}
$$

$$
v_{b} \sim \sum_{n=0}^{\infty} B_{n}^{3} F_{n}\left(t-S^{3}\right),
$$

where the superscripts, $j=1,2,3$ denote the incident, reflected, and transmitted waves, respectively. Since the form for all of the waves is that represented in $\$ 2$, the solution is known provided the initial values $\vec{S}, \vec{A}_{n}^{j}$, and $\vec{B}_{n}^{j}$ are known. It is assumed that the incoming wave has been defined by the methods used above, so that $\bar{S}^{l}$ and $\bar{A}_{n}^{l}$ are known.

From (2), (5) and (8) we can derive the relation

$$
\begin{array}{r}
\rho \gamma B_{n}^{j}=(-1)^{j}\left\{\left(A_{n}^{j+\lambda A_{n-1}^{j}}\right\}-\rho \gamma^{2}\left(B_{n-1}^{j}\right)^{\prime}+(-1)^{j-1} \frac{\rho c^{2} \lambda}{\gamma} B_{n-1}^{j}-\rho c^{2} \lambda\left(B_{n-2}^{j}\right)^{\prime}\right\}, \\
j=1,2,3 .
\end{array}
$$

This relation yields the well-known result from the conservation of momentum across a steadily moving discontinuity, $A_{0}^{j}=\rho \gamma B_{0}^{j}$. Applying the boundary conditions at $x=\bar{x}=L$, we obtain 


$$
\begin{aligned}
& \bar{S}^{1}=\bar{S}^{2}=\bar{S}^{3}, \\
& \vec{A}_{n}^{2}=\bar{A}_{n}^{3}=-\bar{A}_{n}^{1}, \\
& \vec{B}_{n}^{2}-\bar{B}_{n}^{3}=-\bar{B}_{n}^{1} .
\end{aligned}
$$

Now, employing the relation (36) in the third equation in (37), we obtain

$$
\bar{A}_{n}^{2}+\bar{\Gamma} \bar{A}_{n}^{3}=\bar{A}_{n}^{1}-\bar{H}_{n-1}^{\perp}+\bar{H}_{n-2}^{2}+\bar{\Gamma}_{n-1}^{3},
$$

where

$$
H_{n-1}^{j}=-\lambda A_{n-1}^{j}+\rho \gamma^{2}\left(B_{n-1}^{j}\right)^{\prime}+\frac{\rho c^{2} \lambda}{\gamma} B_{n-1}^{j}+\rho c^{2} \lambda\left(B_{n-2}^{j}\right)^{\prime},
$$

and

(40)

$$
\bar{\Gamma}=\bar{\rho}_{a} \bar{\gamma}_{a} / \bar{\rho}_{b} \bar{\gamma}_{b}
$$

Solving the system of equations consisting of the second equation in (37) and $(38)$, we obtain

$$
\begin{aligned}
& \vec{A}_{n}^{2}=\left[(1-\bar{\Gamma}) \bar{A}_{n}^{1}-\bar{H}_{n-1}^{1}+\bar{H}_{n-1}^{2}+\bar{\Gamma} \bar{H}_{n-1}^{3}\right](1+\bar{\Gamma})^{-1}, \\
& \bar{A}_{n}^{3}=\left[2 \bar{A}_{n}^{1}-\bar{H}_{n-1}^{1}+\bar{H}_{n-1}^{2}+\bar{\Gamma} \bar{H}_{n-1}^{3}\right](1+\bar{\Gamma})^{-1} .
\end{aligned}
$$

Thus, since $\bar{S}^{1}$ and $\bar{A}_{n}^{1}$ are known, the initial values at the interface $(x=\vec{x}=L)$ are determined and the above procedures may be used to obtain the formal solution. For example, suppose the end $x=0$ of the semiinfinite bi-viscoelastic rod (which is initially unstressed and at rest) is subjected to a suddenly rising compressive pressure; then to a first term approximation we find that the reflected wave and transmitted wave are given by

(42) $\sigma^{2}(x, t) \sim-\left(\frac{1-\bar{\Gamma}}{1+\bar{\Gamma}}\left[\frac{\gamma_{a}(x) \rho_{a}(x)}{\gamma_{a}(0) \rho_{a}(0)}\right]^{\frac{2}{2}}\right.$

$$
\times \exp \left\{-\frac{1}{2}\left(\int_{0}^{L}-\int_{L}^{x} \frac{\lambda_{a}(\tau)}{\gamma_{a}(\tau)}\left[1-\frac{c_{a}^{2}(\tau)}{\gamma_{\alpha}^{2}(\tau)}\right] d \tau\right\} H\left[t-S^{2}(x)\right],\right.
$$

where 


$$
\left.S^{2}(x)=\iint_{0}^{L}-\int_{L}^{x}\right) \frac{d \tau}{\gamma_{a}(\tau)},
$$

and

$$
\begin{aligned}
& \text { (44) } \sigma^{3}(x, t) \sim-\frac{2 \frac{\tau}{\Gamma^{2}}}{1+\bar{\Gamma}}\left[\frac{\gamma_{b}(x) \rho_{b}(x)}{\gamma_{a}(0) \rho_{a}(0)}\right]^{\frac{1}{2}} \\
& \times \exp \left\{-\frac{1}{2} \int_{0}^{L} \frac{\lambda_{a}(\tau)}{\gamma_{a}(\tau)}\left[1-\frac{c_{a}^{2}(\tau)}{\gamma_{a}^{2}(\tau)}\right] d \tau-\frac{1}{2} \int_{L}^{x} \frac{\lambda_{b}(\tau)}{\gamma_{b}(\tau)}\left[1-\frac{c_{b}^{2}(\tau)}{\gamma_{b}^{2}(\tau)}\right] d \tau\right\} H\left[t-S^{3}(x)\right],
\end{aligned}
$$

where

$$
S^{3}(x)=\int_{0}^{L} \frac{d \tau}{\gamma_{a}(\tau)}+\int_{L}^{x} \frac{d \tau}{\gamma_{b}(\tau)} .
$$

\section{Exact solutions}

In the remaining portion of this paper we shall obtain exact solutions to the partial differential equation (3). We consider the three particular problems solved in $\$ 4$ by the formal Karal-Keller technique. From these exact solutions, wavefront expansions for the stress $\sigma$ are obtained. As will be seen, these expansions agree with those obtained in $\$ 4$ and so provide a verification of the Karal-Keller technique as applied to inhomogeneous viscoelastic bars.

The equation (3) is to be solved subject to the initial conditions

$$
\sigma(x, 0)=\sigma_{t}(x, 0)=\sigma_{t t}(x, 0)=0,
$$

and the boundary condition

$$
\sigma(0, t)=-H(t)
$$

The method of solution will be by means of a Laplace transform

$$
\phi(x, p)=\int_{0}^{\infty} e^{-p t} \sigma(x, t) d t .
$$

On applying (48) to (3), we find that $\phi$ satisfies the ordinary differential equation 


$$
\frac{d^{2 \phi}}{d x^{2}}-r(x) \frac{d \phi}{d x}-\frac{p^{2}[p+\lambda(x)]}{p \gamma^{2}(x)+\lambda(x) c^{2}(x)} \phi=0,
$$

subject to

$$
\phi(0, p)=-\frac{1}{p}
$$

The solution $\sigma$ to the initial-boundary value problem is then recovered from the solution to (49) by using the inverse Laplace transform

$$
\sigma(x, t)=\frac{1}{2 \pi i} \int_{\delta-i \infty}^{\delta+i \infty} e^{p t} \phi(x, p) d p
$$

Here $\delta>0$ is a constant chosen such that $\phi(x, p)$ is an analytic function of $p$ in $\operatorname{Re}(p) \geq \delta$.

It will be the case that the nature of $\sigma(x, t)$ near a front can be discovered by appealing to the asymptotic behavior of $\phi(x, p)$ for large values of the parameter $p$. In connection with this idea we prove the following lemma, which we shall use to extract the wavefront expansion from the integral representation (51) of $\sigma$.

LEMMA. Suppose that $\phi(x, p)$ is analytic in $\operatorname{Re}(p) \geq \delta>0$ and that

$$
\phi(x, p)=\frac{A(x)}{p} \exp \{-p S(x)\}\left[\sum_{n=0}^{N} \frac{A_{n}(x)}{p^{n}}+o\left(\frac{1}{p^{n+1}}\right)\right]
$$

as $p \rightarrow \infty$ in $\operatorname{Re}(p) \geq \delta>0$. Then

(53) $\frac{1}{2 \pi i} \int_{\delta-i \infty}^{\delta+i \infty} e^{p t} \phi(x, p) d p=A(x) H(t-S)\left\{\sum_{n=0}^{N} \frac{A_{n}(x)(t-S)^{n}}{n !}+O\left[(t-S)^{N+1}\right]\right\}$ as $t-S+0$.

Proof. From (52) we may write

(54) $\frac{1}{2 \pi i} \int_{\delta-i \infty}^{\delta+i \infty} e^{p t} \phi(x, p) d p=A(x)\left\{\sum_{n=0}^{N} \frac{A_{n}}{2 \pi i} \int_{\delta-i \infty}^{\delta+i \infty} \exp \{p(t-S)\} \frac{d p}{p^{n+1}}\right.$

$$
\left.+\frac{1}{2 \pi i} \int_{\delta-i \infty}^{\delta+i \infty} \exp \{p(t-S)\} R_{N}(p) d p\right\} \text {, }
$$

where $R_{N}(p)$ is analytic and $O\left(p^{-N-2}\right)$ in $\operatorname{Re}(p) \geq \delta$. 
If $t<S$, then each of the integrals appearing on the right of (54) are zero. This result may be established by closing the contour to the right by means of a large semicircle. There are no singularities in $\operatorname{Re}(p) \geq \delta$ and the integral round the semicircle vanishes as the radius tends to infinity, so the integrals in question are zero.

If $t>S$, we may calculate the first $N$ integrals on the right of (54) by closing the contour to the left. On taking the residue due to the pole at the origin we find that

$$
\frac{1}{2 \pi i} \int_{\delta-i \infty}^{\delta+i \infty} \exp \{(t-S) p\} \frac{d p}{p^{n+1}}=\frac{(t-S)^{n}}{n !}, t>S .
$$

We may also obtain a bound on the last integral appearing in (54). To do so,-split the contour of integration into two pieces denoted by $L_{1}$ and $L_{2}$. On $L_{1},|\operatorname{Im}(p)| \geq \frac{1}{a}$ and on $L_{2},|\operatorname{Im}(p)| \leq \frac{1}{a}$ where $a=t-S$. Then

$$
\left|\int_{L_{1}} \exp \{p(t-S)\} R_{n}(p) d p\right|=0\left(\int_{1 / a}^{\infty} e^{\delta a} \frac{d t}{t^{N+2}}\right)=o\left(a^{N+1}\right),
$$

while if we close $L_{2}$ to the right by means of the semicircle $p=\delta+\frac{1}{a} e^{i \theta}$, where $|\theta| \leq \frac{\pi}{2}$, we get

$$
\left|\int_{L_{2}} \exp \{p(t-S)\} R_{N}(p) d p\right|=o\left[\int_{-\pi / 2}^{\pi / 2} a^{N+1} \exp \left\{a\left(\delta+\frac{1}{a} \cos \theta\right)\right\} d \theta\right]=O\left(a^{N+1}\right)
$$

as $a+0$. Thus, since the integral over the entire contour $\operatorname{Re}(p)=\delta$ vanishes when $a \leq 0$, we may write

$$
\int_{\delta-i \infty}^{\delta+i \infty} \exp \{p(t-S)\} R_{N}(p) d p=H(t-S) O\left[(t-S)^{N+1}\right],
$$

where we have restored the variables $x$ and $t$ according to $a=t-S$. Hence the expressions for the integrals (55) and (56) give equation (53).

\section{Asymptotic results from exact solutions}

We now obtain wave front expansions from the exact solution for each of the problems considered in $\$ 4$. 
PROBLEM 1. Consider the semi-infinite rod whose properties are given by (13). Then inserting these expressions for the coefficients of (49), we find that $\phi$ satisfies

$$
(1+x)^{2} \frac{d^{2 \phi}}{d x^{2}}-r_{0}(1+x) \frac{d \phi}{d x}-\frac{p^{2}\left(p+\lambda_{0}\right)}{\gamma_{0}^{2}\left(p+\lambda_{0} a\right)} \phi=0 .
$$

The change of variable $x+1=e^{z}$ transforms this equation into an equation with constant coefficients. We may then easily discover the general solution to (57). We find that the solution which satisfies the boundary condition (50) and which vanishes as $x \rightarrow \infty$ is

$$
\phi(x, p)=-\frac{1}{p}(x+1)^{\left(x_{0}+1\right) / 2} \exp \left\{-\frac{1}{2} f(p) \ln (x+1)\right\},
$$

where

$$
f(p)=\left(r_{0}+1\right)^{2}+\left[\frac{4 p^{2}\left(p+\lambda_{0}\right)}{\gamma_{0}^{2}\left(p+\lambda_{0} a\right)}\right]^{\frac{1}{2}} .
$$

The branch of the radical which appears in (59) is chosen such that it is positive when $p$ is real, large and positive. With this choice, there exist $\delta>0$ such that $f(p)$ is analytic in $\operatorname{Re}(p) \geq \delta$. In addition, by repeated use of the binomial expansion

$$
f(p)=\frac{2 p}{\gamma_{0}}\left[1+\frac{\gamma_{0}(1-a)}{2 p}+\frac{\beta_{1}}{p^{2}}+\frac{\beta_{2}}{p^{3}}+0\left(\frac{1}{p}\right)\right]
$$

in $\operatorname{Re}(p) \geq \delta$, where $B_{1}, B_{2}$ are given by (22).

According to the lemma of 55, we may discover the behavior of $\sigma(x, t)$ near the wavefront from the asymptotic expansion of $\phi$ for large $p$. On inserting (60) into (58) and using the power series for the exponential function, we get

$$
\begin{aligned}
\phi(x, p)=-\frac{1}{p}(x+1)^{\varepsilon} \exp \{- & \left.\frac{p}{\gamma_{0}} \ln (x+1)\right\}\left\{1-\frac{\beta_{1} \ln (x+1)}{\gamma_{0} p}\right. \\
+ & {\left.\left[\frac{1}{2}\left(\frac{\beta_{1} \ln (x+1)}{\gamma_{0}}\right)^{2}-\frac{\beta_{2} \ln (x+1)}{\gamma_{0}}\right] \frac{1}{p^{2}}+o\left(\frac{1}{p^{3}}\right)\right\}, }
\end{aligned}
$$

where $\varepsilon$ is given by (18). Thus if we apply the inverse Laplace transform 
to $\phi$ and use the result described by (53), we find that

$$
\begin{aligned}
\sigma(x, t)=-(1+x) H(t-S)\{1 & -\frac{\beta_{1} \ln (x+1)}{\gamma_{0}}(t-S) \\
& \left.+\frac{1}{2}\left[\frac{1}{2}\left(\frac{\beta_{1} \ln (x+1)}{\gamma_{0}}\right)^{2}-\frac{\beta_{2} \ln (x+1)}{\gamma_{0}}\right](t-S)^{2}+o\left[(t-S)^{3}\right]\right\},
\end{aligned}
$$

where

$$
S=\frac{1}{\gamma_{0}} \ln (x+1)
$$

This expansion for $\sigma$ is in complete agreement with (21), the expansion obtained by the formal method.

PROBLEM 2. We next consider the impact problem for the finite rod $0 \leq x \leq L$, subject to the boundary condition of vanishing stress at $x=L$. Thus $\phi$, the Laplace transform of $\sigma$, satisfies the equation (49) together with the boundary conditions (50) and

$$
\phi(L, p)=0 \text {. }
$$

We cannot obtain an explicit representation for the solution of the boundary value problem since the coefficients of the differential equation (49) are arbitrary functions of $x$. Nevertheless, we may still verify the results of the Karal-Keller method for this problem. As was seen from the previous problem, the information desired from the solution of the partial differential equation hinged only on the asymptotic behavior of $\phi$ as $p \rightarrow \infty$. We may obtain such asymptotic formulas under mild restrictions on the coefficients of (49), namely differentiability. In particular, we establish the existence of a pair of linearly independent solutions to (49) and obtain the leading term in the asymptotic expansion for large $p$ of each of these solutions. This in turn gives the leading term in the wavefront expansion for the stress $\sigma$.

To determine the behavior of solutions to (49), we follow the methods described in [2]. Make the transformation

$$
z(x)=p \int_{0}^{x} \frac{d \tau}{\gamma(\tau)}+\frac{1}{2} \int_{0}^{x} \frac{\lambda(\tau)}{\gamma(\tau)}\left[1-\frac{c^{2}(\tau)}{\gamma^{2}(\tau)}\right] d \tau,
$$




$$
\phi(x, p)=\left[z^{\prime}(x)\right]^{-\frac{2}{2}} y \exp \left\{\frac{1}{2} \int_{0}^{x} r(\tau) d \tau\right\},
$$

which carries the differential equation (49) into

$$
\frac{d^{2} y}{d z^{2}}-y=\frac{R(x)}{z^{\prime}(x)} y \text {. }
$$

Here, prime denotes differentiation with respect to $x$ and

$$
R(x)=\frac{1}{z^{\prime}}\left\{\frac{d}{d x}\left(\frac{z^{\prime \prime}}{z^{\prime}}-r\right)+\frac{r^{2}}{4}-\left(\frac{z^{\prime \prime}}{z^{\prime}}\right)^{2}+\left(q-z^{\prime 2}\right)\right\},
$$

where

$$
q=\frac{p^{2}(p+\lambda)}{p \gamma^{2}+\lambda c^{2}}
$$

The function $R(x)$ is an analytic function of $p$ which is small when $p$ is large. That is, since

$$
\begin{aligned}
q-z^{\prime 2} & =\frac{\left(p \lambda^{2} c^{2} / \gamma^{2}\right)\left(c^{2} / \gamma^{2}-1\right)}{p \gamma^{2}+\lambda c^{2}}-\frac{\lambda^{2}}{4 \gamma^{2}}\left(\frac{c^{2}}{\gamma^{2}}-1\right) \\
& =O(1)
\end{aligned}
$$

as $p \rightarrow \infty$, then

$$
R(x)=O\left(\frac{1}{p}\right)
$$

uniformly on $0 \leq x \leq L$.

If we apply the method of variation of parameters to (65), temporarily regarding the right hand side as a known function, we obtain the integral equation

$$
y(x)=c_{1} e^{z(x)}+c_{2} e^{-z(x)}+\int_{x_{0}}^{x} \sinh [z(x)-z(\tau)] R(\tau) y(\tau) d \tau,
$$

where $x_{0}$ is some fixed point in $0 \leq x \leq L$, and the $c_{i}^{\prime} s \quad(i=1,2)$ are constants. Equation (69) is a Volterra integral equation; the existence and differentiability of the solution can be demonstrated by constructing the solution by the method of successive approximations. The uniform convergence of this iterative procedure will ensure that the 
solution is an analytic function of $p$ in $\operatorname{Re}(p) \geq 0$, provided the $c_{i}^{\prime} s$ are analytic in $\operatorname{Re}(p) \geq 0$. By differentiating the integral equation, it may be verified that $\phi$ given by $(64)$ satisfies the differential equation (49), where $y$ in (64) is the solution to (69). As well, in view of the bound (68), we may apply standard methods to determine the asynatotic behavior of the solution to the integral equation for any choice of $c_{1}, c_{2}$. Thus in turn we obtain the behavior of solutions to the differential equation.

If we choose $c_{1}=0, c_{2}=1$, and $x_{0}=L$ in (69), we find that (49) has a solution $\phi_{1}(x, p)$ which is analytic in $\operatorname{Re}(p) \geq 0$ and such that

$$
\begin{aligned}
& \phi_{1}(x, p)=\left[\frac{\gamma(x) \rho(x)}{p \rho(0)}\right]^{\frac{1}{2}} e^{-z(x)}\left[1+o\left(\frac{1}{p}\right)\right], \\
& \phi_{1}^{\prime}(x, p)=-\left[\frac{p \rho(x)}{\gamma(x) \rho(0)}\right]^{\frac{1}{2}} e^{-z(x)}\left[1+o\left(\frac{1}{p}\right)\right] .
\end{aligned}
$$

If we choose $c_{1}=1, c_{2}=0$, and $x_{0}=0$, then we obtain a second linearly independent solution $\phi_{2}(x, p)$ which is analytic in $\operatorname{Re}(p) \geq 0$ and such that

$$
\begin{aligned}
& \phi_{2}(x, p)=\left[\frac{\gamma(x) \rho(x)}{p \rho(0)}\right]^{\frac{1}{2}} e^{z(x)}\left[1+o\left(\frac{1}{p}\right)\right], \\
& \phi_{2}^{\prime}(x, p)=\left[\frac{p \rho(x)}{\gamma(x) \rho(0)}\right]^{\frac{1}{2}} e^{z(x)}\left[1+o\left(\frac{1}{p}\right)\right] .
\end{aligned}
$$

Each of these asymptotic formulas holds uniformly in $0 \leq x \leq L$.

We now return to the boundary value problem. The solution to the differential equation (49) which satisfies the boundary conditions (50) and (62) may be expressed in terms the linearly independent pair $\phi_{1}, \phi_{2}$. We find that

$$
\phi(x, p)=\left[\phi_{1}(L, p) \phi_{2}(x, p)-\phi_{2}(L, p) \phi_{1}(x, p)\right] / p \omega(p)
$$

where

$$
\omega(p)=\phi_{1}(0, p) \phi_{2}(L, p)-\phi_{1}(L, p) \phi_{2}(0, p)
$$


this function having the asymptotic behavior

$$
\omega(p)=\frac{2}{P}\left[\frac{\gamma(0) \gamma(L) \rho(L)}{\rho(0)}\right]^{\frac{1}{2}} \sinh \{z(L)\}\left[1+O\left(\frac{1}{p}\right)\right] .
$$

In view of this formula and the fact that

$$
|\sinh \{z(L)\}| \geq A_{0} \exp \{\operatorname{Re}(z(L))\}, \operatorname{Re}(p) \geq \delta>0,
$$

where $A_{0}>0$ is a constant, we may choose $\delta>0$ such that $\omega(p)$ has no zeros in $\operatorname{Re}(p) \geq \delta$.

By applying the inverse Laplace transform to (74), we may obtain the behavior of the stress $\sigma$ near the first incident wavefront and near the first reflected wavefront. This step may be accomplished by first working out the asymptotic form of $\phi$ using the asymptotic formulas for $\phi_{1}, \phi_{2}$, and $\omega(p)$. If we carry out this calculation, applying the identity

$$
(a \sinh z+b \cosh z)^{-1}=\frac{2 e^{-z}}{a+b}+\frac{(a-b) e^{-2 z}}{(a+b)(a \sinh z+b \cosh z)}
$$

to the hyperbolic function appearing in $(76)$, we may write $(74)$ as

$$
\phi(x, p)=\sum_{i=1}^{4} \phi^{i}(x, p)
$$

where

$$
\begin{aligned}
& \phi^{1}(x, p)=-\frac{1}{p}\left[\frac{\gamma(x) \rho(x)}{\gamma(0) \rho(0)}\right]^{\frac{1}{2}} \exp \{-z(x)\}\left[1+O\left(\frac{1}{p}\right)\right], \\
& \phi^{2}(x, p)=\frac{1}{p}\left[\frac{\gamma(x) \rho(x)}{\gamma(0) \rho(0)}\right]^{\frac{1}{2}} \exp \{z(x)-2 z(L)\}\left[1+O\left(\frac{1}{p}\right)\right],
\end{aligned}
$$$$
\phi^{3}(x, p)=\frac{1}{2 p}\left[\frac{\gamma(x) \rho(x)}{\gamma(0) \rho(0)}\right]^{\frac{1}{2}} \frac{\exp \{z(x)-3 z(L)\}}{\sinh \{z(L)\}}\left[1+o\left(\frac{1}{p}\right)\right] \text {, }
$$

$$
\phi^{4}(x, p)=-\frac{1}{2 p}\left[\frac{\gamma(x) \rho(x)}{\gamma(0) \rho(0)}\right]^{\frac{1}{2}} \frac{\exp \{-z(x)-z(L)\}}{\sinh \{z(L)\}}\left[1+0\left(\frac{1}{p}\right)\right] \text {. }
$$

Each of the functions $\phi^{i}$ are analytic functions of $p$ in $\operatorname{Re}(p) \geq \delta$. Thus we have 
(84) $\sigma(x, t)=\sigma^{1}(x, t)+\sigma^{2}(x, t)+\frac{1}{2 \pi i} \int_{\delta-i \infty}^{\delta+i \infty} e^{p t} \phi^{3}(x, p) d p+$ $+\frac{1}{2 \pi i} \int_{\delta-i \infty}^{\delta+i \infty} e^{p t} \phi^{4}(x, p) d p$

where

$$
\begin{aligned}
& \sigma^{1}(x, t)=\frac{1}{2 \pi i} \int_{\delta-i \infty}^{\delta+i \infty} e^{p t} \phi^{1}(x, p) d p, \\
& \sigma^{2}(x, t)=\frac{1}{2 \pi i} \int_{\delta-i \infty}^{\delta+i \infty} e^{p t} \phi^{2}(x, p) d \phi .
\end{aligned}
$$

From the asymptotic formulas for $\phi^{1}, \phi^{2}$ and the definition (63) of $z(x)$, we have on applying the lemma of $\S 5$,

$$
\begin{gathered}
\sigma^{I}(x, t)=-\left[\frac{\gamma(x) \rho(x)}{\gamma(0) \rho(0)}\right]^{\frac{3}{2}} \exp \left\{-\frac{1}{2} \int_{0}^{x} \frac{\lambda(\tau)}{\gamma(\tau)}\left[1-\frac{c^{2}(\tau)}{\gamma^{2}(\tau)}\right] d \tau\right\} H\left(t-S^{1}\right)\left[1+o\left(t-S^{I}\right)\right], \\
S^{\mathcal{I}(x)} \equiv \int_{0}^{x} \frac{d \tau}{\gamma(\tau)},
\end{gathered}
$$

and

$$
\begin{aligned}
& \sigma^{2}(x, t)=\left[\frac{\gamma(x) \rho(x)}{\gamma(0) \rho(0)}\right]^{\frac{1}{2}} \\
& \exp \left\{-\frac{1}{2}\left[\int_{x}^{L}+\int_{0}^{L} \int \frac{\lambda(\tau)}{\gamma(\tau)}\left[1-\frac{e^{2}(\tau)}{\gamma^{2}(\tau)}\right] d \tau\right\} H\left(t-S^{2}\right)\left[1+O\left(t-S^{2}\right)\right],\right. \\
& S^{2}(x) \equiv-\left\{S^{1}(x)-2 S^{1}(L)\right\}=\left[\int_{0}^{L}+\int_{x}^{L}\right\} \frac{d \tau}{\gamma(\tau)} .
\end{aligned}
$$

These formulas are the same as those obtained by the formal method for the first incident and reflected wave. In addition, if we examine the remaining integrals appearing on the right of (84), we find that the first vanishes for $t+S^{l}(x)<4 S^{l}(L)$ and has jumps at $t+S^{l}(x)=2 n S^{l}(L)$, $(n=2,3, \ldots)$, the successive reflected wavefronts; the second is zero for $t-S^{1}(x)<2 S^{\perp}(L)$ and has jumps at $t-S^{\perp}(x)=2 n S^{\perp}(L)$, $(n=1,2, \ldots)$, the successive incident wayefronts. 
PROBLEM 3. We finally examine the biviscoelastic bar, considered in the last of 54, which consists of medium " $a$ " in $0 \leq x \leq L$ and medium " $b$ " in $x \geq L$. For simplicity, we shall assume that medium " $b$ " is homogeneous, that is, that the parameters $\gamma_{b}, r_{b}, c_{b}, \lambda_{b}$ are constants. Since $r=\rho_{x} / \rho$, the density is then assumed to have the form

$$
\rho_{b}(x)=\rho_{b}(L) \exp \left\{r_{b}(x-L)\right\} .
$$

The solution to the transformed equation (49) in $0 \leq x \leq L$, subject to the boundary condition (50); is

$$
\begin{aligned}
\phi_{a}(x, p)=\left[\phi_{1}(0, p) \phi_{2}(x, p)-\phi_{2}(0, p) \phi_{1}(x, p)\right] F(p) / \omega(p) \\
+\left[\phi_{1}(L, p) \phi_{2}(x, p)-\phi_{2}(L, p) \phi_{1}(x, p)\right] / p \omega(p),
\end{aligned}
$$

where

$$
F(p) \equiv \phi(L, p)=\int_{0}^{\infty} e^{-p t} \sigma(L, t) d t
$$

The functions $\phi_{1}, \phi_{2}$, and $\omega(p)$ are the same as in the previous problem except that they are now associated with medium " $a$ ". Thus, the symbols $\gamma, \rho, c$, and $\lambda$, which appear in their asymptotic formulas, should be replaced by $\gamma_{a}, \rho_{a}, c_{a}$, and $\lambda_{a}$.

In medium " $b$ " where $x \geq L$, the equation has constant coefficients. The solution which vanishes as $x \rightarrow \infty$ is

$$
\phi_{b}(x, p)=F(p) \frac{\phi_{3}(x, p)}{\phi_{3}(L, p)},
$$

where

$$
\phi_{3}(x, p) \equiv \exp \left\{\frac{x}{2}\left\{r_{b}-\sqrt{r_{b}^{2}+\frac{4 p^{2}\left(p+\lambda_{b}\right)}{p \gamma_{b}^{2}+\lambda c_{b}^{2}}}\right)\right\} \text {, }
$$

the form of the solution being chosen such that $\phi_{a}(L, p)=\phi_{b}(L, p)$, that is by the boundary condition $\sigma_{a}(L, t)=\sigma_{b}(L, t)$. The branch of the radical which appears in (91) is chosen such that it is real and positive when $p$ is real, large, and positive. It is easy to show that 


$$
\phi_{3}(x, p)=\exp \left\{\frac{1}{2} x\left[r_{b}-\frac{\lambda_{b}}{\gamma_{b}}\left(1-\frac{c_{b}^{2}}{\gamma_{b}^{2}}\right)\right]\right\} e^{-p \frac{x}{\gamma_{b}}}\left[1+o\left(\frac{1}{p}\right)\right] .
$$

The function $F(p)$, which appears in both the expressions for $\phi_{a}$ and $\phi_{b}$, is determined by the remaining boundary condition which requires that $v_{a}(L, t)=v_{b}(L, t)$ where $v(x, t)$ is the particle velocity. Since $u_{t}=v$, where $u$ is the particle displacement, we find from (I) that

$$
p \int_{0}^{\infty} e^{-p t} v(x, t) d t=\frac{1}{\rho(x)} \phi^{\prime}(x, p) .
$$

Thus the boundary condition that $v_{a}(L, t)=v_{b}(L, t)$ implies that

$$
\frac{1}{\rho_{a}(L)} \phi_{a}^{\prime}(L, p)=\frac{1}{\rho_{b}(L)} \phi_{b}^{\prime}(L, p) \text {. }
$$

On using (93) to determine $F(p)$ we get

$$
F(p)=\frac{\rho_{b}(L) \phi_{3}(L, p) \omega_{1}(p)}{p\left[\phi_{3}^{\prime}(L, p) \omega(p) \rho_{a}(L)-\phi_{3}(L, p) \omega_{2}(s) p_{b}(L)\right]},
$$

where

$$
\begin{aligned}
& \omega_{1}(p)=\phi_{1}(L, p) \phi_{2}^{\prime}(L, p)-\phi_{1}^{\prime}(L, p) \phi_{2}(L, p), \\
& \omega_{2}(p)=\phi_{1}(0, p) \phi_{2}^{\prime}(L, p)-\phi_{2}(0, p) \phi_{1}^{\prime}(L, p) .
\end{aligned}
$$

The function $F(p)$ has the asymptotic behavior

$$
F(p)=-\frac{1}{p} \frac{\left[\gamma_{a}(L) \rho_{a}(L) / \gamma_{a}(0) \rho_{a}(0)\right]^{\frac{1}{2}}}{\cosh \{z(L)\}+\bar{\Gamma} \sinh \{z(L)\}}\left[1+0\left(\frac{1}{p}\right)\right]
$$

in $\operatorname{Re}(p) \geq \delta>0$, where

$$
\bar{\Gamma}=\rho_{a}(L) \gamma_{a}(L) / \rho_{b}(L) \gamma_{b}(L)
$$

Since the linear combination of hyperbolic functions in (95) satisfies an analogous inequality to $(77)$, we may choose $\delta>0$ such that $F(p)$ has no singularities in $\operatorname{Re}(p) \geq \delta$.

To discover the behavior of $\sigma$, it remains only to look at each of 
the solutions $\phi_{a}, \phi_{b}$ for large $p$. Consider $\phi_{a}$ first. Using the identity $(78)$ in connection with the hyperbolic function appearing in the asymptotic formula $(76)$ for $\omega(p)$ and with those hyperbolic functions in (95), we find the following information about each of the terms appearing in (88):

$$
\begin{aligned}
\phi_{1}(0, p) \phi_{2}(x, p) F(p) / \omega(p) & =\Phi_{1}(x, p)+\Phi_{2}(x, p), \\
\phi_{2}(0, p) \phi_{1}(x, p) F(p) / \omega(p) & =\Phi_{3}(x, p), \\
\phi_{1}(L, p) \Phi_{2}(x, p) / p \omega(p) & =\Phi_{4}(x, p)+\Phi_{5}(x, p), \\
\phi_{2}(L, p) \phi_{1}(x, p) / p \omega(p) & =\Phi_{6}(x, p)+\Phi_{7}(x, p),
\end{aligned}
$$

where each of the functions $\Phi_{i}$ is analytic in $\operatorname{Re}(p) \geq \delta$ and has the asymptotic behavior

$$
\begin{aligned}
& \Phi_{1}(x, p)=-\frac{2}{p}\left[\frac{\gamma_{a}(x) \rho_{a}(x)}{\gamma_{a}(0) \rho_{a}(0)}\right] \frac{\exp \{z(x)-2 z(L)\}}{1+\bar{\Gamma}}\left[1+0\left(\frac{1}{p}\right)\right], \\
& \Phi_{2}(x, p)=0\left[\frac{1}{p} \exp \{z(x)-4 z(L)\}\right], \\
& \Phi_{3}(x, p)=0\left[\frac{1}{p} \exp \{-z(x)-2 z(L)\}\right], \\
& \Phi_{4}(x, p)=\frac{1}{p}\left[\frac{\gamma_{a}(x) \rho_{a}(x)}{\gamma_{a}(0) \rho_{a}(0)}\right]^{\frac{1}{2}} \exp \{z(x)-2 z(L)\}\left[1+0\left(\frac{1}{p}\right)\right], \\
& \Phi_{5}(x, p)=\frac{1}{2 p}\left[\frac{\gamma_{a}(x) \rho_{a}(x)}{\gamma_{a}(0) \rho_{a}(0)}\right]^{\frac{1}{2}} \frac{\exp \{z(x)-3 z(L)\}}{\sinh \{z(L)\}}\left[1+o\left(\frac{1}{p}\right)\right], \\
& \Phi_{6}(x, p)=\frac{1}{p}\left[\frac{\gamma_{a}(x) \rho_{a}(x)}{\gamma_{a}(0) \rho_{a}(0)}\right]^{\frac{1}{2}} \exp \{-z(x)\}\left[1+o\left(\frac{1}{p}\right)\right], \\
& \Phi_{7}(x, p)=\frac{1}{2 p}\left[\frac{\gamma_{a}(x) \rho_{a}(x)}{\gamma_{a}(0) \rho_{a}(0)}\right]^{\frac{1}{2}} \frac{\exp \{-z(x)-z(L)\}}{\sinh \{z(L)\}}\left[1+o\left(\frac{1}{p}\right)\right] .
\end{aligned}
$$

The function $z(x)$, which appears in each of these formulas, is the same as before, namely, 


$$
z(x)=p S^{1}(x)=\frac{1}{2} \int_{0}^{x} \frac{\lambda_{a}(\tau)}{\gamma_{a}(\tau)}\left[1-\frac{c_{a}^{2}(\tau)}{\gamma_{a}^{2}(\tau)}\right] d \tau,
$$

where

$$
S^{I}(x)=\int_{0}^{x} \frac{d \tau}{\gamma_{a}^{(\tau)}}
$$

Now apply the inverse Laplace transform to $\phi_{a}$, using the above information to write the stress as the sum of several terms in the following way:

$$
\sigma_{a}(x, t)=\sigma^{1}(x, t)+\sigma^{2}(x, t)+\sigma_{a}^{r}(x, t),
$$

where

(96) $\sigma^{I}(x, t)=-\frac{1}{2 \pi i} \int_{\delta-i \infty}^{\delta+i \infty} e^{p t} \Phi_{6}(x, p) d p$,

(97) $\sigma^{2}(x, t)=\frac{1}{2 \pi i} \int_{\delta-i \infty}^{\delta+i \infty} e^{p t}\left[\Phi_{1}(x, p)+\Phi_{4}(x, p)\right] d p$,

(98) $\sigma_{a}^{r}(x, t)=\frac{1}{2 \pi i} \int_{\delta-i \infty}^{\delta+i \infty} e^{p t}\left[\Phi_{2}(x, p)+\Phi_{5}(x, p)\right] d p-$

$$
-\frac{1}{2 \pi i} \int_{\delta-i \infty}^{\delta+i \infty} e^{p t}\left[\Phi_{3}(x, p)+\Phi_{7}(x, p)\right] d p \text {. }
$$

Examining each of these expressions reveals that the only contribution to the stress due to the first incident and reflected wave comes from $\sigma^{l}$ and $\sigma^{2}$. The expression (96) for $\sigma^{l}$ gives the same result as in the previous problem for the behavior of the stress near the first incident wave. Similarly, examining $\sigma^{2}$ gives the behavior of the stress near the first reflected front. From the asymptotic formula for the integrand in (97) we find that 


$$
\begin{aligned}
\sigma^{2}(x, t)= & -\left[\frac{\gamma_{a}(x) \rho_{a}(x)}{\gamma_{a}(0) \rho_{a}(0)}\right]^{\frac{1}{2}} \\
& \times \exp \left\{-\frac{1}{2}\left(\int_{0}^{L}+\int_{x}^{L} \frac{\lambda_{a}(\tau)}{\gamma_{a}^{(\tau)}}\left[1-\frac{c_{a}^{2}(\tau)}{\gamma_{a}^{2}(\tau)}\right] d \tau\right\} H\left(t-S^{2}\right)\left[\frac{1-\bar{\Gamma}}{1+\bar{\Gamma}}+\theta\left(t-S^{2}\right)\right],\right.
\end{aligned}
$$

where

$$
\left.S^{2}(x)=-\left\{S^{\perp}(x)-2 S^{\perp}(L)\right\}=\iint_{0}^{L}+\int_{x}^{L}\right\} \frac{d \tau}{\gamma_{a}(\tau)} .
$$

Looking at $\sigma_{a}^{r}$, we find that this quantity is identically zero during the time that the disturbance first leaves the end $x=0$ until it next reaches this end. From the behavior of the integrand, the first integral appearing in (98) vanishes for $t+S^{l}(x)<4 S^{l}(L)$. It has jumps at $t+S^{l}(x)=2 n S^{1}(L) \quad(n=2,3, \ldots)$, the successive fronts reflected from the end $x=L$. Similarily the second integral appearing in (98) vanishes for $t-S^{l}(x)<2 S^{l}(L)$ and has discontinuities at $t-S^{l}(x)=2 n S^{l}(L)$ $(n=1,2, \ldots)$, the remaining wavefronts incident on $x=L$.

Finally we look at medium " $b$ ". In a similar fashion as for medium " $\alpha$ ", we may write (90) as

$$
\Phi_{b}(x, p)=\Phi_{b}^{1}(x, p)+\Phi_{b}^{r}(x, p),
$$

where

$$
\begin{aligned}
\Phi_{b}^{l}(x, p)=-\frac{2}{p} \frac{\bar{\Gamma}^{\frac{1}{2}}}{1+\bar{\Gamma}} & {\left[\frac{\rho_{b}(x) \gamma_{b}}{\gamma_{a}(0) \rho_{a}(0)}\right]^{\frac{1}{2}} } \\
& \times \exp \left\{-\frac{1}{2} \frac{\lambda_{b}}{\lambda_{b}}\left[1-\frac{c_{b}^{2}}{\gamma_{b}^{2}}\right)(x-L)-\frac{p}{\gamma_{b}}(x-L)-z(L)\right\}\left[1+o\left(\frac{1}{p}\right)\right],
\end{aligned}
$$

and

$$
\Phi_{b}^{r}(x, p)=0\left[\frac{1}{p} \exp \left\{-\frac{p}{\gamma_{b}}(x-L)-3 z(L)\right\}\right] .
$$

Then, the stress $\sigma_{b}(x, t)$ has the form 


$$
\sigma_{b}(x, t)=\sigma^{3}(x, t)+\sigma_{b}^{r}(x, t),
$$

where

$$
\begin{aligned}
\sigma_{b}^{r}(x, t) & =\frac{1}{2 \pi i} \int_{\delta-i \infty}^{\delta+i \infty} e^{p t} \Phi_{b}^{r}(x, p) d p \\
& =0 \text {, for } t-\frac{1}{\gamma_{b}}(x-L)-3 S^{l}(L)<0,
\end{aligned}
$$

and

$$
\begin{aligned}
\sigma^{3}(x, t)= & \frac{1}{2 \pi i} \int_{\delta-i \infty}^{\delta+i \infty} e^{p t_{\Phi} I}(x, p) d p \\
& =\frac{2 \overrightarrow{\Gamma^{2}}}{1+\bar{\Gamma}}\left[\frac{\rho_{b}(x) \gamma_{b}}{\gamma_{a}(0) \rho_{a}(0)}\right]^{\frac{1}{2}} H\left(t-S^{3}\right) \\
& \exp \left\{-\frac{1}{2} \frac{\lambda_{b}}{\gamma_{b}}\left[1-\frac{c_{b}^{2}}{\gamma_{b}^{2}}\right)(x-L)-\frac{1}{2} \int_{0}^{L} \frac{\lambda_{a}(\tau)}{\gamma_{a}(\tau)}\left[1-\frac{c_{a}^{2}(\tau)}{\gamma_{a}^{2}(\tau)}\right] d \tau\right\}\left[1+O\left(t-S^{3}\right)\right],
\end{aligned}
$$

where

$$
S^{3}(x)=S^{1}(L)+\frac{1}{\gamma_{b}}(x-L)=\int_{0}^{L} \frac{d \tau}{\gamma_{a}^{(\tau)}}+\frac{1}{\gamma_{b}}(x-L) .
$$

These formulas for the bi-viscoelastic problem are in agreement with those obtained in $\$ 4$, where in comparing the last expansion with that obtained by the formal method, it should be remembered that the parameters which appear in connection with medium " $b$ " were assumed constant.

\section{Discussion}

We have presented the formal Karal-Keller asymptotic technique as it applies to propagation of Iongitudinal waves in viscoelastic bars. Our primary purpose was to provide justifications for the Karal-Keller technique as well as demonstrating that this formal method provides a direct way of solving asymptotically wave propagating problems governed by hyperbolic partial differential equations. In the first part of this paper, the Karal-Keller technique was used to solve several problems of propagation, reflection, and transmission of impact waves. In the second part, the same problems were solved by means of the Laplace transform. For 
each of the problems considered, the asymptotic formulas obtained by the transform method were in complete agreement with those obtained by the formal method, thus providing several verifications of the Karal-Keller technique.

The Karal-Keller method is a direct procedure which involves only ordinary differentiation, integration, and algebra. Because of its directness, it is invariably true that it is much simpler than the procedure of 56 , which involves first finding the exact solution, and then its asymptotic expansion.

At the present time no general proof of the validity of the formal method has been published. It is therefore important to show, for problems which can be solved by other means, that the solutions give results which agree with the asymptotic formulas obtained by the Karal-Keller method.

\section{References}

[1] Henry F. Cooper, Jr., "Propagation of one-dimensional waves in inhomogeneous elastic media", SIAM Rev. 9 (1967), 671-679.

[2] A. Erdélyi, Asymptotic expansions (Dover, New York, 1956).

[3] F.G. Friedlander, "Simple progressive solutions of the wave equation", Proc. Cambridge Philos. Soc. 43 (1967), 360-373.

[4] Frank C. Karal, Jr., and Joseph B. Keller, "Elastic wave propagation in homogeneous and inhomogeneous media", J. Acoust. Soc. Amer. 31 (1959), 694-705.

[5] H.A. Lang, "Two verifications of the Karal-Keller theory of wave propagation", J. Acoust. Soc. Amer. 34 (1962), 785-788.

[6] Peter D. Lax, "Asymptotic solutions of oscillatory initial value problems", Duke Math. J. 24 (1957), 627-646.

[7] Robert M. Lewis and Joseph B. Keller, Asymptotic methods for partial differential equations; the reduced wave equation and Maxwell's equations (Report EM-194, Courant Institute of Mathematical Sciences, New York University, New York, 1964). 
[8] Donald Ludwig, "Uniform asymptotic expansions for wave propagation and diffraction problems", STAM Rev. 12 (1970), 325-331.

[9] R.K. Luneburg, Mathematical theory of optics (University of California Press, Berkeley, Los Angeles, 1964).

[10] T. Bryant Moodie, "On the propagation of radially symetric waves in nonhomogeneous isotropic elastic media", Utilitas Math. 2 $(1972), 181-203$.

[11] T. Bryant Moodie, "Cylindrical and spherical shear waves in nonhomogeneous isotropic viscoelastic-media", Canad. J. Phys. 51 (1973), 1091-1097.

[12] T. Bryant Moodie, "On the propagation, reflection, and transmission of transient cylindrical shear waves in nonhomogeneous fourparameter viscoelastic media", Bull. Austral. Math. Soc. 8 (1973), 397-411.

[13] Im K. Park, Edward L. Reiss, "Oscillatory impact of an inhomogeneous viscoelastic rod", J. Acoust. Soc. Amer. 47 (1970), 870-874.

Department of Mathematics,

University of Alberta,

Edmonton,

Alberta,

Canada. 